\title{
CARACTERÍSTICAS CLIINICAS DEL CÁNCER DE TIROIDES EN MANIZALES, COLOMBIA, 2008-2015
}

\author{
Nelson E. Arias-Ortiz (iD),a , Eduardo A. Guzmán-Gallego iid²,a \\ 1 Grupo de investigación en Promoción de la Salud y Prevención de la Enfermedad, Universidad de Caldas, Manizales, \\ Colombia. \\ 2 Universidad de Caldas, Manizales, Colombia, \\ a Médico cirujano
}

\section{RESUMEN}

Con el objetivo de describir los casos de cáncer de tiroides en Manizales, Colombia, se realizó un estudio que describe las características de los pacientes y de los tumores según el tamaño, la lateralidad, la focalización, el compromiso ganglionar, la invasión contigua, la metástatisis y la estadificación TNM. Se analizaron 672 casos, el 84,8\% de ellos fueron mujeres entre 40 y 64 años. El 34,1\% de los casos fueron diagnosticados en estadios tempranos y el $15 \%$ de los tumores tuvieron un tamaño $<20 \mathrm{~mm}$. El compromiso ganglionar y de tejidos adyacentes estuvo presente en el 33\% y el 3\% de los casos, respectivamente. Se registró un $1 \%$ de metástasis a distancia. El carcinoma papilar se presentó en el $82 \%$ de los casos. El cáncer de tiroides en Manizales es más frecuente en mujeres adultas; el estadio temprano y el tamaño tumoral sugieren mejora en la detección temprana.

Palabras clave: Cáncer; Neoplasias de la tiroides; Epidemiologia (fuente: DeCS BIREME).

\section{CLINICAL CHARACTERISTICS OF THYROID CANCER IN MANIZALES, COLOMBIA, 2008-2015}

\begin{abstract}
A study was conducted to describe the cases of thyroid cancer in Manizales, Colombia. This study describes the characteristics of patients; and tumors according to size, laterality, focalization, nodal involvement, contiguous invasion, metastasis and TNM staging. A total of 672 cases were analyzed, $84.8 \%$ of which were women between 40 and 64 years of age. From the population, 34.1\% were cases diagnosed in early stages and $15 \%$ of the tumors were $>20 \mathrm{~mm}$ in size. Nodal and adjacent tissue involvement was present in 33\% and 3\% of the cases, respectively. Distant metastasis was documented in $1 \%$ of the cases. Papillary carcinoma was present in $82 \%$ of cases. Thyroid cancer in Manizales is more frequent in adult women. Tumor size and being at the early stages are factors that suggest improvement in early detection.
\end{abstract}

Keywords: Cancer; Thyroid neoplasms; Epidemiology (source: MeSH NLM).

\section{INTRODUCCIÓN}

El cáncer de tiroides (CT) es la enfermedad endocrina maligna más común y deriva de células epiteliales, como la papilares, foliculares, anaplásicas y medulares en el $90 \%$ de los casos. En

Citar como: Arias-Ortiz NE, Guzmán-Gallego EA. Características clínicas del cáncer de tiroides en Manizales, Colombia, 2008-2015. Rev Peru Med Exp Salud Publica. 2020;37(2):287-91. doi: https://doi. org/10.17843/rpmesp.2020.372.4892

Correspondencia: Eduardo A. Guzmán-Gallego, eduardo.guzman@ ucaldas.edu.co

Recibido: 18/10/2019

Aprobado: $15 / 04 / 2020$

En línea: 16/06/2020 el mundo, se ubica en el puesto dieciséis entre los cánceres más frecuentes, con un $2 \%$ del total de casos nuevos ${ }^{(1)}$. En varios países latinoamericanos, esta neoplasia está entre las primeras diez en frecuencia en ambos sexos, siendo mucho más frecuente en mujeres ${ }^{(2)}$. Colombia cuenta con datos de incidencia de base poblacional en cinco ciudades según las estimaciones nacionales de incidencia de cáncer a partir de la mortalidad ${ }^{(3,4)}$. Las últimas incidencias estimadas fueron de 16,0 y de 0,7 por 100000 en mujeres y hombres, respectivamente, lo que ubica al CT como el tercero más común en mujeres luego de mama y cuello uterino ${ }^{(1)}$.

Manizales, capital del departamento de Caldas, es una ciudad andina colombiana con cerca de 430000 habitantes ${ }^{(5)}$ y cuenta con un registro de cáncer de base poblacional indexado ante la Agencia Internacional para la Investigación en Cáncer (IARC, por sus siglas en inglés). Este registro realiza la búsqueda activa de casos nuevos de neoplasias malignas en la población residente en zonas urbana y rural del municpio; las fuentes de información provie- 
nen de hospitales y clínicas, centros de imagenología y endoscopía, laboratorios clínicos y laboratorios de histopatología, además de información de mortalidad oficial. El registro es operado por la Universidad de Caldas, en convenio con el Instituto Nacional de Cancerología de Colombia y cumple con los estándares de calidad recomendados por la IARC ${ }^{(6)}$. Sus datos de incidencia han sido publicados en los volúmenes 10 y 11 de Cancer Incidence in Five Continents, publicación de referencia mundial en epidemiología descriptiva del cáncer. Según datos de este registro, la incidencia de CT pasó de 12,3 y 3,2 por 100000 en mujeres y hombres, respectivamente, en el periodo 2003-2007 a 23,2 y 4,6 en el quinquenio 2008-2012, lo que representa un aumento porcentual anual promedio de $13,4 \%$ y $7 \%$, respectivamente ${ }^{(2)}$. Estas tasas son significativamente superiores a las reportadas por otras ciudades con registro poblacional en el país: para mujeres y hombres, respectivamente, Bucaramanga reportó 14,4 y 2,5; Cali 13,5 y 3,2; y Pasto 11,8 y 2,6 por 100000 habitantes ${ }^{(2)}$.

El objetivo de esta investigación fue describir las características de los casos de CT en Manizales, Colombia durante el periodo 2008-2015.

\section{EL ESTUDIO}

Investigación observacional, descriptiva, retrospectiva, transversal, basada en datos de base poblacional. Se recopiló la información del total de pacientes incidentes de CT (código topográfico C73X de la Clasificación Internacional para Enfermedades en Oncología, tercera edición primera revisión, CIEO3.1) entre el 1 de enero de 2008 y el 31 de diciembre de 2015, sin aplicar criterios de exclusión. Se revisaron reportes histopatológicos con el fin ampliar las descripciones microscópicas de los tumores.

Como variables del paciente se describieron el sexo y la edad, como variables del tumor se revisaron el tipo histológico, número de lesiones, tamaño, lateralidad, compromiso de tejidos adyacentes y de ganglios linfáticos, y diseminación a distancia; a partir de la combinación de estas variables se describió el estadio clínico al momento del diagnóstico con base en el sistema TNM de la American Joint Committee on Cancer (AJCC), 8. a edición. Para clasificar los tumores según su tamaño, se usó el punto de corte de $20 \mathrm{~mm}$ para clasificar lesiones pequeñas sugerido por Herránz González-Botas J, Barro CV y Vidal JM ${ }^{(7,8)}$.

Se calcularon medianas y rangos intercuartílicos para describir las tendencias de las variables numéricas. Para las variables categóricas se presentan frecuencias absolutas y relativas. Para probar las posibles diferencias de las características de los tumores según sexo se utilizó la prueba $U$ de Mann-Whitney (suma de rangos) y la prueba de Chi cuadrado contenida en el paquete estadístico Stata versión $14.2 \circledast$, dado que las variables siguen distribuciones diferentes a la normal.

\section{MENSAJES CLAVE}

Motivación para realizar el estudio: El cáncer de tiroides es la enfermedad endocrina maligna más común y deriva de células epiteliales.

Principales hallazgos: El 82\% de los casos fueron carcinomas papilares en estadios tempranos, predominantemente en mujeres de 40 a 65 años, y de un tamaño $<20$ mm.

Implicancias: Los estadios tempranos y tamaños pequeños sugieren mejoría en el diagnóstico temprano. El predominio en mujeres, el posible efecto cohorte y el aumento a expensas del carcinoma papilar, sugieren aumento real de la incidencia.

El proyecto de investigación fue aprobado por el Comité de Bioética de la Universidad de Caldas y clasificado como investigación sin riesgo de acuerdo con la normatividad vigente en Colombia (Resolución 8430 de 1993, art. 11).

\section{HALLAZGOS}

Se analizaron 672 casos incidentes. La mediana de edad al diagnóstico fue 51,1 años y rango intercuartílico (RIQ) 21,4 años. Los grupos etáreos más comprometidos van de los 40 a los 64 años, con predominio de 45 a 49 años. El sexo femenino predominó con el $84,8 \%$ del total de casos $(n=570)$ y una razón mujer/hombre de 5:1. La histología predominante fue el carcinoma papilar con el 81,7\% de los casos.

La mayoría de los casos (85\%) correspondieron a tumores pequeños (<20 mm); sin embargo, hubo un $40 \%$ de casos con dato perdido para esta variable. Se encontraron lesiones más grandes en los hombres, en comparación con las mujeres $(p=0,004)$. El tamaño de los carcinomas papilares fue aparentemente más pequeño que el de los demás tipos histológicos. Los carcinomas no especificados tuvieron también un tamaño menor, lo que sugiere que podrían corresponder a carcinomas papilares que no fueron histológicamente verificados. Las características de los casos de cáncer de tiroides según sexo se presentan en la Tabla 1.

Se encontró predominio de tumores unifocales (50,2\%), unilaterales $(52,4 \%)$ y compromiso ganglionar en una tercera parte de los casos (ganglios positivos 33\%, 60\% en el grupo anatómico III). Solo el 3,2\% de los casos presentaron invasión a laringe, tráquea, y faringe; las metástasis distantes se presentaron en el 1,0\% de los casos.

Respecto al estadio clínico-patológico de presentación de la enfermedad, se encontraron datos completos para estadificación en cerca del $60 \%$ de los casos, de los cuales el $82,6 \%$ corresponden a estadio I y el $12,4 \%$ al estadio II. 
Tabla 1. Características de los casos de cáncer de tiroides en Manizales, Colombia, 2008-2015

\begin{tabular}{|c|c|c|c|c|c|}
\hline \multirow{2}{*}{ Característica } & \multicolumn{2}{|c|}{ Hombres } & \multicolumn{2}{|c|}{ Mujeres } & \multirow[b]{2}{*}{ Valor de $\mathrm{p}^{\mathrm{b}}$} \\
\hline & $\mathbf{n}$ & $\%$ & $\mathbf{n}$ & $\%$ & \\
\hline Edad $^{\text {a }}$ & 55,9 & $45,0-66,1$ & 50,5 & $40,1-61,3$ & 0,020 \\
\hline Tamaño en $\mathrm{mm}^{\mathrm{a}}$ & 19 & $10-40$ & 13 & $8-20$ & 0,004 \\
\hline \multicolumn{6}{|l|}{ Lateralidad } \\
\hline Unilateral & 48 & 47,0 & 304 & 53,3 & 0,733 \\
\hline Bilateral & 8 & 7,8 & 44 & 7,7 & \\
\hline Sin dato & 46 & 45,1 & 222 & 38,9 & \\
\hline \multicolumn{6}{|l|}{ Focos tumorales } \\
\hline Único & 43 & 42,2 & 294 & 51,6 & 0,241 \\
\hline Múltiple & 12 & 11,8 & 54 & 9,5 & \\
\hline Sin dato & 47 & 46,1 & 222 & 38,9 & \\
\hline \multicolumn{6}{|l|}{ Compromiso ganglionar } \\
\hline Presente & 33 & 32,3 & 166 & 29,1 & 0,309 \\
\hline Ausente & 29 & 28,4 & 193 & 33,9 & \\
\hline Sin dato & 40 & 39,2 & 211 & 37,0 & \\
\hline Número de ganglios ${ }^{a}$ & 4,2 & $0-24$ & 2,2 & $0-47$ & 0,038 \\
\hline \multicolumn{6}{|c|}{ Compromiso de tejidos adyacentes } \\
\hline Sí & 4 & 3,9 & 18 & 3,2 & 0,558 \\
\hline No & 53 & 52,0 & 333 & 58,4 & \\
\hline Sin dato & 45 & 44,1 & 219 & 38,4 & \\
\hline \multicolumn{6}{|l|}{ Metástasis } \\
\hline Sí & 1 & 1,0 & 6 & 1,04 & 0,941 \\
\hline No & 53 & 52,0 & 345 & 60,5 & \\
\hline Sin dato & 48 & 47,1 & 219 & 38,4 & \\
\hline Total & 102 & 15,2 & 570 & 84,8 & \\
\hline
\end{tabular}

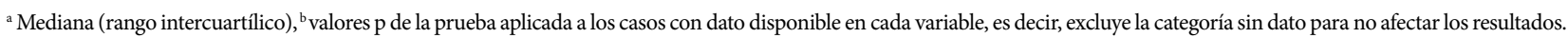

\section{DISCUSIÓN}

Este estudio describe las características de los casos de CT en Manizales (Colombia) y señala que su mayor frecuencia es en mujeres, en grupos de edad entre 40 y 64 años, con lesiones pequeñas diagnosticadas en estadio temprano y con predominio del carcinoma papilar. Hubo diferencias por sexo a favor de las mujeres en la mediana de edad al momento del diagnóstico, en la mediana del tamaño tumoral y en el número de ganglios comprometidos.

La distribución de casos por grupos de edad encontrada es consistente con un estudio en Cataluña (España) y con una serie de casos reportada por Chala y colaboradores, en Manizales (Colombia) ${ }^{(9,10)}$. La razón de feminidad de 5:1, la predominancia del tipo histológico papilar y la diferencia de tamaño promedio de los tumores entre hombres y mujeres encontradas en este estudio concuerdan con lo reportado por Herránz, $\mathrm{J}^{(7)}$.

Con relación a la estadificación al momento del diagnóstico, la mayoría de los pacientes estuvieron en etapas tempranas del cáncer de tiroides, cifra que es casi el doble de lo reportado en La Coruña, España, donde el $47 \%$ de los pacientes de 45 y más años fueron estadio $I^{(7)}$. Este resultado apoyaría la hipótesis de un fenómeno de sobrediagnóstico en la población de Manizales.

Varios informes han descrito un aumento espectacular de la incidencia del cáncer de tiroides en las últimas décadas, predominantemente carcinomas papilares pequeños, aun cuando las tasas de mortalidad relacionadas con el cáncer de tiroides no han cambiado en forma sustancial ${ }^{(1,11)}$. Este fenómeno ha sido observado en diferentes poblaciones del mundo, especialmente en mujeres. Aunque no son completamente claras las razones de este incremento, se le atribuye a la mejor y mayor utilización de las técnicas diagnósticas, especialmente de la ecografía de tejidos blandos del cuello ${ }^{(9)}$. El aumento de la incidencia es más marcado en las mujeres y se concentra en el grupo de edad de 45 a 49 años, lo cual podría estar relacionado con la mayor frecuencia de uso de los servicios de salud por mujeres de estas edades en comparación con los hombres. 
Manizales podría no ser ajena a este fenómeno de incremento en la incidencia de CT. De acuerdo con datos previos del Registro Poblacional de Cáncer de Manizales, la incidencia de CT en mujeres en este municipio se duplicó entre los quinquenios 2003-2007 y 2008-2012 y pasó a ocupar el segundo lugar en frecuencia de todas las neoplasias malignas en mujeres; también se observó un incremento importante de la incidencia en hombres ${ }^{(2)}$.

Algunos autores han postulado que el mejoramiento de la detección temprana, asociado a la mejora en las técnicas de diagnóstico, y el mayor acceso a la atención médica por parte de la población son la explicación más plausible para el incremento notorio de la incidencia. Entre los argumentos expuestos en la literatura se encuentran la difusión de procedimientos médicos avanzados, como el ultrasonido; la incidencia aumentada de microcarcinomas; el aumento de diagnósticos incidentales (tiroidectomías por enfermedades benignas; mayor detalle de los estudios anatomo-patológicos, el descubrimiento incidental de nódulos tiroideos durante otros procedimientos, como ecografías carotídeas); la alta frecuencia de cánceres tiroideos pequeños y asintomáticos en autopsias; $y$ una mayor exhaustividad en los registros de cáncer ${ }^{(12-16)}$.

Sin embargo, algunos estudios señalan que dicho aumento se explica tanto por la mejora en los servicios de salud, como por un aumento real en la incidencia ${ }^{(15-17)}$. Los argumentos que apoyan esta controversia son el aumento simultáneo de tumores grandes; la falta de descenso en la incidencia de tumores de mayor tamaño y en etapa avanzada como resultado de una supuesta mejora en la detección temprana; el aumento predominante del tipo histológico papilar y no de todos los tipos como se esperaría a consecuencia de la mejora en el diagnóstico; la distribución no uniforme del aumento de la incidencia por edad y sexo (predominio en mujeres y presencia de efecto cohorte); la falta de aumento de la incidencia para otros tumores como resultado de una supuesta mayor exhaustividad de los registros de cáncer; y la falta de descenso de la mortalidad como producto de una supuesta mayor detección temprana, y por lo contrario, su aumento leve sobretodo en hombres.

Por otro lado, el aumento concentrado en sexo femenino y en ciertos grupos de edad (posible efecto cohorte) podría también ser explicado por exposiciones diferenciales entre hombres y mujeres a factores de riesgo no conocidos, por ejemplo, a sustancias con potencial comportamiento como disruptores endocrinos y con posibles efectos inductores de carcinogénesis tiroidea ${ }^{(18,19)}$. El aumento casi exclusivamente a expensas del tipo histológico papilar también puede ser interpretado como argumento a favor de un posible aumento real de la incidencia combinado con aumento artificial por mejora en el diagnóstico ${ }^{(20-22)}$. Además, el predominio de tumores de tamaño inferior a $2 \mathrm{~cm}$ y en estadios tempranos, apoya también conclusiones en este sentido.

Una fortaleza del presente estudio fue el hecho de trabajar con datos de base poblacional con estándares de calidad internacional ${ }^{(6)}$ indexados en la IARC ${ }^{(2)}$. No obstante, se observaron limitaciones importantes en la completitud de datos para algunas variables del tumor que no son de recolección rutinaria por parte del registro. Los resultados del presente estudio sirven de base para nuevas investigaciones que exploren la exposición a posibles factores de riesgo mediante diseños más complejos. Surge también la posibilidad de estudiar la posible asociación entre carcinomas de tiroides y el uso masivo de la ecografía como método de estudio inicial que favorecería el sobrediagnóstico de la enfermedad en la población.

En conclusión, en Manizales el CT afectó principalmente a las mujeres. El tipo histológico papilar fue más frecuente en mujeres que en hombres. $\mathrm{Al}$ momento del diagnóstico, los estadios tempranos fueron predominantes. Hubo diferencias por sexo a favor de las mujeres en la mediana de edad al momento del diagnóstico, en la mediana del tamaño tumoral y en el número de ganglios comprometidos. Algunas de las características de los pacientes y de los tumores hacen plausible la hipótesis de un escenario de sobrediagnóstico cuya magnitud deberá ser tema de futuras investigaciones.

Fuentes de financiamiento: Instituto Nacional de Cancerología de Colombia y la Universidad de Caldas, Colombia.

Conflictos de interés: Los autores declaran no tener conflictos de intereses en la publicación del artículo.

Contribuciones de autoría: NEAO y EAGG han participado en la concepción del artículo, la recolección de datos, la redacción y aprobación de la versión final.

\section{REFERENCIAS BIBLIOGRÁFICAS}

1. Castro-Jiménez MA, López-Daza DF. Estabilidad de la mortalidad por cáncer de tiroides en un país emergente. Rev Salud Pública. 2015; 17 (1): 1-11. doi: http://dx.doi.org/10.15446/rsap.v17n1.42209.

2. Bray F, Colombet M, Mery L, Piñeros M, Znaor A, Zanetti R, et al. Cancer Incidence in Five Continents Volume XI [Internet]. 2017 [citado el 2 de noviembre de 2017]. Disponible en: http://ci5.iarc. fr/CI5-XI/PDF/BYSITE/C73.pdf.
3. Uricoechea HV, Chaparro JH, Cabrera IM, Delgado VA. Epidemiología del cáncer de tiroides. Análisis de resultados en Sudamérica y Colombia. Rev Med. 2015;37(2):140-63.

4. Pardo C. Cendales R. Incidencia, mortalidad y prevalencia de Cáncer en Colombia 20072011. Bogotá, D. C.: Instituto Nacional de Cancerología; 2015. $148 \mathrm{p}$.

5. DANE. Población ajustada por cobertura censal. Censo General de Población y Vivienda. 2018. 
6. Arias-Ortiz N, López-Guarnizo G. Evaluación de Calidad de los Datos del Registro Poblacional de Cáncer de Manizales, Colombia. Rev Colomb Cancerol. 2013;17(4):13241.

7. Herránz González-Botas J, Barro CV, Vidal JM. Grupos de riesgo en carcinomas diferenciados de tiroides. Acta Otorrinolaringol Esp. 2011;62(1):149. doi: 10.1016/j.otorri.2010.09.007.

8. Zhi J, Wu Y, Hu L, Zhao J, Liu H, Ruan X, et al. Assessment of the prognostic value and N1b changes of the eighth TNM/AJCC staging system for differentiated thyroid carcinoma. Int J Clin Oncol. 2020. 25(1):59-66. doi: 10.1007/s10147-019-01522-x.

9. Zafon C, Puig-Domingo M, Biarnés J, Halperin I, Bella MR, Castells I, et al. Estudio descriptivo de las características del cáncer diferenciado de tiroides en Cataluña en el periodo 1998-2012. Registro CECaT. Endocrinol Nutr. 2015;62(6):2649. doi: 10.1016/j.endonu.2015.03.005.

10. Chala A, Franco H, Aguilar C, Cardona J. Estudio descriptivo de doce años de cáncer de tiroides, Manizales, Colombia. Rev Colomb Cir. 2010;(25):27689.

11. Kilfoy BA, Zheng T, Holford TR, Han X, Mary H, Sjodin A, et al. International patterns and trends in thyroid cancer incidence, 1973-2002. Cancer Causes Control. 2009;20(5):525-31. doi: 10.1007/s10552-008-9260-4.

12. Vaccarella S, Franceschi S, Bray F, Wild CP, Plummer M, Dal Maso L. Worldwide Thyroid-Cancer Epidemic? The Increasing Impact of Overdiagnosis. N Engl J Med. 2016;375(7):6147. doi: 10.1056/NEJMp1604412.

13. Morris LGT, Sikora AG, Tosteson TD, Davies L. The Increasing Incidence of Thyroid Cancer: The Influence of Access to Care. Thyroid. 2013;23(7):88591. doi: 10.1089/thy.2013.0045.

14. Hoang JK, Nguyen XV, Davies L. Overdiagnosis of Thyroid Cancer: Answers to Five Key Questions. Acad Radiol. 2015;22(8):10249. doi: 10.1016/j.acra.2015.01.019.

15. Li N, Du XL, Reitzel LR, Xu L, Sturgis EM. Impact of enhanced detection on the increase in thyroid cancer incidence in the United States: review of incidence trends by socioeconomic status within the surveillance, epidemiology, and end results registry, 1980-2008. Thyroid. 2013;23(1):10310. doi: 10.1089/thy.2012.0392.

16. Enewold LR, Zhou J, Devesa SS, Gonzalez AB De, Marrogi AJ, Potter JF, et al. Thyroid cancer incidence among active duty U.S military personnel, 1990-2004. Cancer Epidemiol Biomarkers Prev. 2011;20(11):236976. doi: 10.1158/1055-9965.EPI-11-0596.

17. Pellegriti G, Frasca F, Regalbuto C, Squatrito S, Vigneri R. Worldwide increasing incidence of thyroid cancer: update on epidemiology and risk factors. J Cancer Epidemiol. 2013;2013:965212. doi: $10.1155 / 2013 / 965212$.

18. Arias-Ortiz NE, Icaza-Noguera G, Ruiz-Rudolph P. Thyroid cancer incidence in women and proximity to industrial air pollution sources: A spatial analysis in a middle size city in Colombia. Atmos Pollut Res. 2018;9(3):46475.

19. Arias-Ortiz NE, Ruiz-Rudolph P. Potential thyroid carcinogens in atmospheric emissions from industrial facilities in Manizales, a midsize Andean city in Colombia. Atmos Pollut Res. 2017;8(6):105868.

20. Morris LGT, Myssiorek D. Improved detection does not fully explain the rising incidence of well-differentiated thyroid cancer: a population-based analysis. Am J Surg. 2010;200(4):45461. doi: 10.1016/j. amjsurg.2009.11.008.

21. Chala AI, Pava R, Franco HI, Álvarez A, Franco A. Criterios ecográficos diagnósticos de neoplasia maligna en el nódulo tiroideo: correlación con la punción por aspiración con aguja fina y la anatomía patológica. Rev Colomb Cir. 2013;(28):1523.

22. Manso García S, Velasco Marcos MJ. Valor actual de la ecografía en la caracterización de los nódulos tiroideos. Revisión de las últimas guías clínicas de actuación. Radiología. Mayo de 2015;57(3):24858. doi: 10.1016/j.rx.2014.03.001. 